\title{
Effects of a prosody rehabilitation program on expression of affect in preschool children with hearing impairment: a randomized trial
}

\author{
Ossama A. Sobhy ${ }^{1}$, Rania M. Abdou², Sara M. Ibrahim² ${ }^{*}$ (D) and Nesrine H. Hamouda²
}

\begin{abstract}
Background: Children with hearing impairment are deprived of their source of linguistic input which in turn leads to linguistic and prosodic deficits that negatively affect language and social development. Linguistic aspects other than prosody have received considerable attention in studies concerned with hearing-impaired children with little literature addressing how to improve their affective prosodic deficits. The aim of the current study is to adapt and apply the "prosody treatment program" and detect the effect of prosodic rehabilitation on affect production and language development in Egyptian hearing-impaired children. This study was conducted on 21 children with sensorineural hearing loss. The subjects were randomly divided into two groups, group A (cases) and group B (control) by block randomization. The subjects of the study were evaluated pre and post-therapy by a protocol for assessment of their prosodic skills using subjective and objective measures. Both groups received the usual auditory and language rehabilitation therapy. The case group additionally received rehabilitation for prosody using the "prosody treatment program" for 3 months.

Results: Results showed a statistically significant improvement in the subjective scores and most of the objective scores of the assessed affective prosodic skills when comparing pre-therapy and post-therapy scores in the cases group, and when comparing both studied groups post-therapy.

Conclusions: Prosodic training has an additional benefit evident in improving affective prosodic skills of hearingimpaired children compared to conventional therapy alone with a positive effect on their linguistic development.

Trial registration: The trial is registered at www.clinicaltrials.gov NCT04691830. Registered under the name "Adaptation of a Rehabilitation Program for Prosody and its Application on Egyptian Hearing Impaired Children". Retrospectively registered:
\end{abstract}

Keywords: Prosody, Rehabilitation, Hearing impairment, Cochlear implant, Auditory rehabilitation

\section{Background}

Prosody is the melody and rhythm of speech. It represents the suprasegmental aspects of speech in the form of pitch, loudness, and duration. Each language has its unique phonological system and prosodic characteristics.

\footnotetext{
* Correspondence: s_ibrahim16@alexmed.edu.eg

2Unit of Phoniatrics, Department of Otorhinolaryngology, Faculty of

Medicine, Alexandria University, Champollion Street, Alexandria 21131, Egypt

Full list of author information is available at the end of the article
}

The main prosodic forms are conveyed as prosodic cues which are intonation, stress, and rhythm and these components vary among languages [1]. Pitch, loudness, and duration are conveyed by their acoustic correlates including fundamental frequency (f0), intensity, and the length of the acoustic intervals respectively [2].

Prosody has several communicative functions such as its affective, pragmatic, and grammatical functions [3]. Affective prosody involves the manipulation of prosodic

\section{Springer Open}

() The Author(s). 2021 Open Access This article is licensed under a Creative Commons Attribution 4.0 International License, which permits use, sharing, adaptation, distribution and reproduction in any medium or format, as long as you give appropriate credit to the original author(s) and the source, provide a link to the Creative Commons licence, and indicate if changes were made. The images or other third party material in this article are included in the article's Creative Commons licence, unless indicated otherwise in a credit line to the material. If material is not included in the article's Creative Commons licence and your intended use is not permitted by statutory regulation or exceeds the permitted use, you will need to obtain permission directly from the copyright holder. To view a copy of this licence, visit http://creativecommons.org/licenses/by/4.0/. 
features to convey the attitude of the speaker allowing communicative partners to share their feelings and incorporate register variations to accommodate different social contexts [4-6]. Processing of affect requires both auditory and visual information; as a result, hearingimpaired individuals are at a disadvantage. Hearingimpaired children are deprived of auditory input early in life resulting in a negative impact on language and speech development. Early language stimulation paves the way to social and emotional development. Studies indicated that hearing-impaired children with adequate language have fewer psychological and social challenges compared to their peers with more delayed language [7]. Moreover, adequate exposure of cochlear implanted (CI) children to spoken language is reported to be associated with better social welfare [8].

Fundamental frequency (f0) variations are the main cues responsible for conveying different emotions in speech and music. Studies demonstrated that CI children usually perform poorly in emotion recognition tasks when compared to normal hearing children and this poor performance in emotional recognition has been connected to poor perception of pitch cues $[9,10]$. Previous research showed that moderate to profound hearing-impaired children demonstrate inadequate perception of emotion in auditory only, visual only, and auditory-visual conditions [11]. This concludes that pitch perception is challenging, not only for CI users but also for those using hearing aids (HAs).

Several suprasegmental deficits are characteristic of the speech of the hearing-impaired particularly prosodic control of intonation and stress [12, 13]. Regarding affective prosody production, some studies showed that $\mathrm{CI}$ children produce emotions with a limited range of fo and intensity with little contrasts between different emotions in addition to an imprecise imitation of emotions due to inappropriate pitch inflection and an overall monotonous speech $[14,15]$.

Prosody remediation programs for hearing impairment include speech training programs, sensory feedback programs, and Music training.

Speech training programs aim at providing activities targeting auditory discrimination, imitation, and eliciting appropriate speech production. Klieve and Jeanes [16] applied an intervention program that included activities focusing on tone and affect, questions and statements, intensity, stress, chunking, and grammar. The program activities highlighted meaning distinctions delivered by speech prosody.

Friedman [17] trained three severe to profound hearing-impaired children in auditory training, imitation, and production of terminal declination of pitch slope at the end of utterances to elicit appropriate production of terminal F0 in utterances.
Sensory feedback programs utilizing sensory aids in addition to auditory feedback are based on the need for an additional aid to provide feedback due to poor perception of f0 changes by hearing-impaired individuals.

Computer-based training programs have been beneficial in improvement of prosodic aspects of HI children. A computer-based Hungarian prosody teaching system was created by David Stazho, GaborKiss, and Klara Visci [18] to provide a visual display on loudness, intonation (displayed in the form fundamental frequency variation over time), and rhythm for hearing impaired individuals.

Several studies addressed the role of music and rhythm training in improving the prosodic skills of hearing impaired children, particularly the perception of stress patterns, F0 contours, and music perception [19-21]

Studies focusing on prosodic intervention in hearingimpaired children have not received much attention as other speech and language aspects. Most of the previously published interventions were non-comprehensive and targeted a restricted range of prosodic skills.

In light of the role of prosody in social interaction and maturity, the current study aims at examining the effect of rehabilitation of prosody using the adapted translated version of the "prosody treatment program" [22] on Arabic-speaking Egyptian hearing-impaired children, particularly its role in the improvement of affect production and its implication on language development in comparison to the conventional auditory and language training alone.

\section{Methods \\ Study design}

Participants were enrolled in two parallel groups in a randomized controlled study design. The study was designed to assess the effect of training by the "prosody treatment program" [22] on the expression of affect in pre-school age children with hearing impairment in comparison to traditional auditory and language rehabilitation alone. The research was conducted in the time interval between February 2019 and February 2020 after approval of the ethics committee (IRB No: 00012098). The study adheres to CONSORT guidelines.

\section{Study participants}

Subjects included in the study were 21 hearing-impaired children of both sexes, in the pre-school age (from 3 to 6 years). Children were randomly divided by a randomization block design into a case group $(\mathrm{n}=11)$ which received the "prosody treatment program" [22] in addition to the usual auditory and language rehabilitation provided at the phoniatrics unit, and a control group $(\mathrm{n}=10)$ which received the usual rehabilitation without prosodic intervention.

Inclusion criteria of the study participants were preschool age children (3-6 years) using auditory-verbal 
communication with severe up to profound sensorineural hearing loss (SNHL) in unaided conditions, fitted with HAs or CIs, with satisfactory benefit (aided hearing threshold less than $40 \mathrm{~dB}$ across all frequencies). Exclusion criteria included children with intellectual disability, brain damage, additional sensory impairment, psychiatric comorbidities (example; autism spectrum disorder), and children with motor speech disorders such as apraxia of speech. Data were collected and the study was conducted at the Phoniatrics Unit, Department of Otorhinolaryngology.

\section{Interventions}

The "Prosody Treatment Program" [22] was translated to Arabic and used for prosodic training of Egyptian pre-school age children with hearing impairment. The program was applied to group A (case group) in individual sessions, one session per week, each session was about $1 \mathrm{~h}$ in duration. Both groups received the usual auditory and language rehabilitation in thirty-minute sessions, twice per week for 3 months.

\section{Translation of the "Prosody Treatment Program" [22]}

The program was translated to Arabic and necessary modifications were done to adapt it to the Arabic language and Egyptian culture after permission from the publisher. The translated version was revised by three experienced bilingual phoniatricians.

\section{The "Prosody Treatment Program" [22]}

The principles of validated interventions are implemented in the program such as Melodic Intonation Therapy (MIT), Lee Silverman Voice Treatment (LSVT), and Dynamic Temporal and Tactile Cueing (DTTC) for Speech Motor Learning, in addition to expert professional practice [23-25]. The program is divided into two sections according to the age into preschool section and school age section. Only the pre-school section was used in this study. Activities included in the program target the main prosodic features; pitch, loudness, and duration and their implementation for adequate production of different prosodic components such as stress and intonation.

\section{Prosodic skills targeted in the program}

The program included the following activities and materials to practice basic prosodic skills.

- Musical instruments: to help increase the child's awareness of the concept of pitch and rhythm and provide the opportunity to experiment with aspects of rate, loudness, and different melodic patterns. In this activity, the clinician modeled simple patterns and encouraged the child to imitate them (e.g., quiet-quiet-loud). Appropriate feedback was given throughout the activity such as "That was nice and slow" and "That was really fast."

- Vocalizing: helped the child practice producing vocal inflections. An example is the "Ahhhh song" which focused on vocalization and pitch without using words, only the vowel /a/ was used. This activity is appropriate for children with minimal verbal expression.

- Animal sounds: helped children to manipulate their voices using fun activities. In the "Guess who I am" activity, the clinician and children cut out pictures of different animals. The clinician and the child took turns modeling the sound and guessing the animal.

- Expressions: included activities that encouraged the child to use frequent daily used expressions produced with increased inflection. An example in the pretend cooking activity using toy food and kitchen items. A silly food was prepared such as dirty sock soup, the child was encouraged to imitate the clinician's expressions such as "Ewww, gross" in contrast to making a tasty meal and using expressions such as "mmmm" or "yummy."

- First sentences: this activity included three songs provided with picture cues for each song. The songs contained common phrases produced with typical vocal stress and speech inflection. One of the songs is the "it's your turn, it's my turn" song which targeted language and turn taking by singing the song using stress on the pronoun by saying it using a higher pitch, loudness, and duration in each phrase with the use of picture cues.

- Quiet/whisper voice: included playing a game to help the child practice quiet/whisper voice.

- Character voices: included a story provided with pictures with different characters using distinct voices for each character (such as a high-pitched voice for the baby bear) to help the child practice different vocal tones.

All activities were provided with tips to help the clinician apply the activity properly and provide feedback to the child.

The following expressive task cues were implemented starting with the most independent level and moving back a step till the child was successful and training proceeded towards achieving independent responses in the most advanced level. The cues are based on the principles of DTTC [25].

Expressive task cues:

1. The child says the utterance in unison with you.

2. The child repeats the utterance while watching your face. 
3. The child repeats the utterance without watching your face.

4. The child repeats the utterance without watching your face, after a 2-3-s delay.

5. The child says the utterance independently without any cues.

\section{Auditory and language rehabilitation}

Auditory rehabilitation included activities targeting auditory skills: sound detection, discrimination, identification, and auditory comprehension. Language rehabilitation included activities aiming at improving semantics, syntax, pragmatics, and phonology. The training for both studied groups was administered by the same phoniatrician.

\section{Study outcomes}

The primary outcome of the study was the assessment of the affective prosodic skills of hearing-impaired children after the intervention, and this was done using items of the Prosodic skill assessment protocol assessing affect [26]. The secondary outcomes of the study were the assessment of the effect of rehabilitation on the language and cognitive development of the participants using the Arabic language test and Stanford-Binet Scale, respectively $[27,28]$.

\section{Assessment}

All children were subjected to the following protocol of assessment before and after intervention:

1- History taking including personal data, complaint, analysis of symptoms, and detailed present, past, and family history.

2- General examination, neurological examination, and oral motor examination.

3- Audiological evaluation by pure tone audiometry to determine aided and un-aided hearing threshold levels.

4- Psychometric evaluation by Stanford-Binet Scale 4th edition [28] to assess verbal intelligence quotient, abstract intelligence quotient, and general intelligence quotient.

5- Arabic language test [27] to assess receptive language, expressive language, semantics, pragmatics, and prosody. The child's score was then compared to the mean of his/her age group to determine the child's language age.

6- Evaluation of prosody by prosodic skill assessment protocol [26].

\section{Subjective assessment}

The assessment protocol originally included the assessment of ten prosodic skills: five affective and five grammatical skills. Only the five subtests assessing affective prosodic skills were used for this study:

1- Expression of anger: To elicit this pattern each participant was shown pictures illustrating an anger scene, for example, a picture of a mother expressing her anger towards her daughter who has broken a toy saying/ ænæ zæ læ:næ mennek/

2- Verbal disapproval phrases: elicited by showing pictures representing a disapproval act, for example, a picture of a child pulling on her sister's hair and her mother disapproving of what she is doing and telling her that this is wrong. /kedæ Yalat/

3- Verbal exclamation, to elicit the surprise prosodic pattern by showing pictures representing an exclamation scene, for example, the picture of a girl who has just received a new dress and is saying /Ralla:h fUstae:n gæmi:1/

4- Wishful pattern elicited by showing pictures demonstrating wishful pattern, such as, a picture of a girl studying and wishing that she will succeed and saying /ja rab ængæh/

5- Warning patterns: to elicit the warning prosodic pattern from each child, each child was shown pictures to evoke this pattern, such as a picture of a girl putting her hand near a knife and her mother warning her that this is dangerous by saying / Rew i Pelsekinæ tegræhek/

The child had to produce sentences with the appropriate stress in order to obtain a full score for the tested item. Each skill was assessed using four pictures, the first and the second pictures were used as a demo and a trial respectively. The demo and the trial pictures were not scored. The third and fourth pictures represented the test stimuli to evoke sentence production with appropriate stress patterns.

The results were numerically scored for the third and fourth pictures as follows:

0 - Incorrect or no response.

1- One correct response with help (imitation).

2- Two correct responses with help (imitation).

3- One correct spontaneous response + another correct response with help (imitation).

4- Two correct responses spontaneously with $100 \%$ accuracy.

Subjective scores were obtained from two phoniatricians with a minimum of 3 years of experience, separately and blindly, to ensure the reliability of the subjective assessment.

\section{Objective analysis}

The five prosodic skills assessed by the prosodic skill assessment protocol were recorded and analyzed by a 
spectrogram, a program of computerized speech lab (CSL) model 4500-Kay Pentax to obtain the following data from each utterance from its beginning to its end:

1. The mean pitch contour in $\mathrm{Hz}$.

2. The mean energy contour representing the energy in $\mathrm{dB}$.

3. Mean duration of the sound wave of the whole utterance, calculated in seconds.

\section{Randomization and blinding}

The allocation sequence was generated using permuted block randomization technique and the block size was four participants per block. The allocation sequence was concealed from the person allocating the participants to the intervention arms using sealed opaque envelopes by the statistician. A double-blinded approach was adopted. Blinding was employed on participants and outcome assessors who were blinded to the group allocation of patients.

\section{Statistical methodology}

Data were analyzed using IBM SPSS software package version 20.0. (Armonk, NY: IBM Corp). Qualitative data were described using number and percent. The Kolmogorov-Smirnov test was used to verify the normality of distribution. Quantitative data were described using range (minimum and maximum), mean, standard deviation, median, and Interquartile range (IQR). Significance of the obtained results was judged at the $5 \%$ level.

Chi-square test was used to test the association between qualitative variables. Fisher's exact test and Monte Carlo corrections were carried out when indicated $(n \times$ $m$ table or expected cells less than 5).

Comparisons were carried out between two studied independent normally distributed subgroups using Student's $t$ test. Comparisons were carried out between two studied related normally distributed subgroups using paired $t$ test. Comparisons were carried out between two studied independent not-normally distributed subgroups using Mann-Whitney U test. Comparisons were carried

Table 1 Comparison between the two studied groups according to demographic data and history

\begin{tabular}{|c|c|c|c|c|c|c|}
\hline & \multicolumn{2}{|c|}{ Group $A(n=11)$} & \multicolumn{2}{|c|}{ Group $B(n=10)$} & \multirow{2}{*}{$\begin{array}{l}\text { Test of } \\
\text { significance }\end{array}$} & \multirow[t]{2}{*}{$P$ value } \\
\hline & No. & $\%$ & No. & $\%$ & & \\
\hline \multicolumn{7}{|l|}{ Sex } \\
\hline Male & 3 & 27.3 & 2 & 20.0 & $x^{2}=0.153$ & $\mathrm{FE}_{p}=1.000$ \\
\hline Female & 8 & 72.7 & 8 & 80.0 & & \\
\hline \multicolumn{7}{|c|}{ Age pre-intervention (years) } \\
\hline Mean $\pm S D$ & $5.11 \pm 0.94$ & & $4.94 \pm 0.59$ & & $t=0.473$ & 0.642 \\
\hline \multicolumn{7}{|c|}{ Age of diagnosis of $\mathrm{HI}$ (years) } \\
\hline Mean \pm SD & $1.63 \pm 1.24$ & & $1.28 \pm 0.89$ & & $t=0.743$ & 0.466 \\
\hline \multicolumn{7}{|c|}{ Timing of hearing loss } \\
\hline Pre-lingual & 10 & 90.9 & 9 & 90.0 & $x^{2}=0.005$ & $\mathrm{FE}_{p}=1.000$ \\
\hline Peri-lingual & 1 & 9.1 & 1 & 10.0 & & \\
\hline \multicolumn{7}{|l|}{ Degree of SNHL } \\
\hline Bilateral profound & 9 & 81.8 & 10 & 100.0 & $x^{2}=1.881$ & ${ }^{\mathrm{MC}} p=1.000$ \\
\hline Bilateral severe & 1 & 9.1 & 0 & 0.0 & & \\
\hline Severe-profound & 1 & 9.1 & 0 & 0.0 & & \\
\hline \multicolumn{7}{|l|}{$\mathrm{HA} / \mathrm{Cl}$} \\
\hline $\mathrm{Cl}$ & 8 & 72.7 & 9 & 90.0 & $x^{2}=1.014$ & ${ }^{\mathrm{FE}} p=0.586$ \\
\hline $\mathrm{HA}$ & 3 & 27.3 & 1 & 10.0 & & \\
\hline \multicolumn{7}{|l|}{ Hearing age (years) } \\
\hline Mean \pm SD & $1.75 \pm 1.21$ & & $1.51 \pm 1.28$ & & $t=0.444$ & 0.662 \\
\hline \multicolumn{7}{|c|}{ Previous rehabilitation } \\
\hline No & 1 & 9.1 & 1 & 10.0 & $x^{2}=0.005$ & ${ }^{\mathrm{FE}} p=1.000$ \\
\hline Yes & 10 & 90.9 & 9 & 90.0 & & \\
\hline
\end{tabular}

X2 chi-squared test, FE Fisher's exact, MC Monte Carlo, $t$ Student's t test, $p p$ value for comparing between the studied groups, Group A cases, Group B control Pre-lingual hearing impairment: hearing impairment before language acquisition, either congenital or in early childhood.

Peri-lingual hearing impairment: hearing impairment when some language has been acquired, before complete language development.

Hearing age: the duration since satisfactory amplification or cochlear implantation 
out between two studied related not-normally distributed subgroups using Wilcoxon signed-rank test. An alpha level was set to $5 \%$ with a significance level of $95 \%$, and a beta error accepted up to $20 \%$ with a power of study of $80 \%$ (when calculating the sample size). The sample size was calculated using two proportions power analysis in NCSS \& PASS program.

Intraclass correlation coefficient was used for the agreement between rater 1 and rater 2 for subjective assessment of the studied preschool age groups.

\section{Results}

The 21 participants were analyzed, group A cases $(\mathrm{n}=$ $11)$, and group $B$ control $(n=10)$ after a period of 3 months of intervention which was the predetermined period of time necessary to complete the intervention.

Descriptive analysis, $t$ test and chi-square test showed that the two groups were matching regarding their demographic data and relevant history (Table 1).

There was a statistically significant increase in verbal IQ $(p=0.038)$ of the cases group when comparing pretherapy and post-therapy scores. No statistically significant difference was found in abstract IQ and general IQ. When both studied groups were compared post-therapy, no statistically significant difference was found (Table 2)

Regarding the Arabic language test, the mean difference between pre-therapy and post-therapy scores of both groups was compared. The results showed a statistically significant improvement in the pragmatic language age $(p=0.046)$ and the total language age $(p=$ 0.032 ) in the case group (Table 3 ).

\section{Subjective assessment of prosody}

Comparison between pre-therapy and post-therapy subjective assessment scores showed a statistically significant increase in all subjective scores among the preschool cases group (group A). When comparing pretherapy and post-therapy scores of the control group, no statistically significant difference was found in any of the studied skills. When both group post-therapy scores were compared, a statistically significant difference was found in all of the assessed patterns (Table 4).

Subjective scores of the cases and control group pre and post-intervention were rated separately and blindly by two raters. Intraclass correlation coefficient showed that most of the scores had between good (ICC between 0.75 and 0.90 ) to excellent agreement (above 0.90) denoting reliability of the subjective assessment (Table 5).

\section{Objective prosodic assessment}

Mean F0, mean intensity, and mean duration were measured for each utterance, as these parameters were found to be among the most important ones signaling emotional prosody in previous studies [29]. The following results were obtained from the acoustic analysis of the five assessed utterances:

- Anger pattern, verbal disapproval, verbal exclamation, and wishful thinking showed a statistically significant increase in duration between pre-therapy and post-therapy in the cases group.Expression of anger is reported in literature to be associated with an increase

Table 2 Comparison between the two studied groups according to Stanford-Binet Scale

\begin{tabular}{|c|c|c|c|c|c|}
\hline & Stanford-Binet & Group $A(n=11)$ & Group $B(n=10)$ & Test of significance & $P$ value \\
\hline \multirow[t]{5}{*}{ Verbal IQ } & Pre-therapy & & & & \\
\hline & Mean \pm SD & $64.73 \pm 20.75$ & $64.0 \pm 25.60$ & $t=0.072$ & 0.943 \\
\hline & Post-therapy & & & & \\
\hline & Mean \pm SD & $75.27 \pm 11.77$ & $67.70 \pm 20.24$ & $t=1.061$ & 0.302 \\
\hline & $p_{1}$ & $0.038^{*}$ & 0.445 & & \\
\hline \multirow[t]{5}{*}{ Abstract IQ } & Pre-therapy & & & & \\
\hline & Mean $\pm S D$ & $93.36 \pm 8.14$ & $92.70 \pm 10.38$ & $t=0.164$ & 0.872 \\
\hline & Post-therapy & & & & \\
\hline & Mean \pm SD & $92.36 \pm 8.46$ & $92.20 \pm 9.17$ & $t=0.043$ & 0.967 \\
\hline & $p_{1}$ & 0.772 & 0.676 & & \\
\hline \multirow[t]{5}{*}{ General IQ } & Pre-therapy & & & & \\
\hline & Mean \pm SD & $76.27 \pm 10.48$ & $75.0 \pm 18.99$ & $t=0.193$ & 0.849 \\
\hline & Post therapy & & & & \\
\hline & Mean \pm SD & $81.45 \pm 9.63$ & $75.80 \pm 14.33$ & $t=1.071$ & 0.298 \\
\hline & $p_{1}$ & 0.059 & 0.804 & & \\
\hline
\end{tabular}

$t$ Student's t-test, $p p$ value for comparing between the studied groups, $p_{1} p$ value for paired test for comparing between pre and post therapy, Group $A$ cases, Group B control 
Table 3 Comparison between the two studied groups according to mean difference of Arabic language test

\begin{tabular}{|c|c|c|c|c|}
\hline Mean difference of Arabic language test & Group $A(n=11)$ & Group $B(n=10)$ & U & $\mathrm{p}$ \\
\hline \multicolumn{5}{|l|}{ Semantic language age (years) } \\
\hline Median (IQR) & $0.50(0.0-1.25)$ & $0.25(0.0-1.50)$ & 48.0 & 0.654 \\
\hline \multicolumn{5}{|l|}{ Receptive language age } \\
\hline Median (IQR) & $1.0(0.50-1.0)$ & $0.75(0.0-1.0)$ & 32.50 & 0.104 \\
\hline \multicolumn{5}{|l|}{ Expressive language age } \\
\hline Median (IQR) & $0.50(0.50-1.0)$ & $0.25(0.0-1.0)$ & 41.0 & 0.304 \\
\hline \multicolumn{5}{|l|}{ Pragmatic age } \\
\hline Median (IQR) & $1.0(0.25-1.25)$ & $0.0(0.0-0.50)$ & $28.50^{*}$ & $0.046^{*}$ \\
\hline \multicolumn{5}{|l|}{ Prosody age } \\
\hline Median (IQR) & $1.0(0.75-1.75)$ & $1.0(0.0-1.50)$ & 43.50 & 0.406 \\
\hline \multicolumn{5}{|l|}{ Total language age } \\
\hline Median (IQR) & $1.0(0.501 .75)$ & $0.50(0.0-0.50)$ & $26.0^{*}$ & $0.032^{*}$ \\
\hline
\end{tabular}

U Mann-Whitney test, IQR interquartile range, $p p$ value for comparing between the studied groups, Group A cases, Group $B$ control

*Statistically significant at $p \leq 0.05$

Table 4 Comparison between the two studied groups according to subjective assessment of prosodic skills

\begin{tabular}{|c|c|c|c|c|c|}
\hline \multicolumn{2}{|c|}{ Subjective assessment of prosodic skills } & \multirow[t]{2}{*}{ Group $A(n=11)$} & \multirow[t]{2}{*}{ Group B(n = 10) } & \multirow[t]{2}{*}{ Test of sig. } & \multirow[t]{2}{*}{$p$} \\
\hline Anger pattern & Pre-therapy & & & & \\
\hline & Mean \pm SD & $1.64 \pm 1.12$ & $1.30 \pm 1.06$ & $U=45.0$ & 0.453 \\
\hline & Post-therapy & & & & \\
\hline & Mean \pm SD & $2.82 \pm 1.08$ & $1.70 \pm 0.95$ & $U=25.0^{*}$ & $0.025^{*}$ \\
\hline & $p_{1}$ & $0.010^{*}$ & 0.102 & & \\
\hline \multirow[t]{5}{*}{ Verbal disapproval } & Pre-therapy & & & & \\
\hline & Mean \pm SD & $2.45 \pm 1.37$ & $1.80 \pm 0.92$ & $U=42.0$ & 0.343 \\
\hline & Post-therapy & & & & \\
\hline & Mean \pm SD & $3.27 \pm 0.79$ & $1.70 \pm 0.48$ & $U=7.0^{*}$ & $<0.001^{*}$ \\
\hline & $p_{1}$ & $0.030^{*}$ & 0.655 & & \\
\hline \multirow[t]{5}{*}{ Verbal exclamation } & Pre-therapy & & & & \\
\hline & Mean \pm SD & $2.09 \pm 1.22$ & $2.20 \pm 0.63$ & $U=53.50$ & 0.911 \\
\hline & Post-therapy & & & & \\
\hline & Mean \pm SD & $3.36 \pm 0.81$ & $2.20 \pm 0.92$ & $U=19.50^{*}$ & $0.009^{*}$ \\
\hline & $p_{1}$ & $0.016^{*}$ & 1.000 & & \\
\hline \multirow[t]{5}{*}{ Wishful thinking } & Pre-therapy & & & & \\
\hline & Mean \pm SD & $2.18 \pm 1.08$ & $1.80 \pm 1.23$ & $U=47.50$ & 0.574 \\
\hline & Post-therapy & & & & \\
\hline & Mean \pm SD & $3.36 \pm 0.92$ & $1.80 \pm 1.03$ & $U=13.50^{*}$ & $0.002^{*}$ \\
\hline & $p_{1}$ & $0.026^{*}$ & 1.000 & & \\
\hline \multirow[t]{6}{*}{ Warning speech } & Pre-therapy & & & & \\
\hline & Mean \pm SD & $2.27 \pm 1.19$ & $1.30 \pm 0.48$ & $U=29.0^{*}$ & $0.046^{*}$ \\
\hline & Post-therapy & & & & \\
\hline & Mean \pm SD & $3.18 \pm 0.87$ & $1.80 \pm 0.63$ & $U=13.50^{*}$ & $0.002^{*}$ \\
\hline & Mean difference & $\uparrow 0.91 \pm 1.14$ & $\uparrow 0.50 \pm 0.71$ & $U=44.0$ & 0.406 \\
\hline & $p_{1}$ & $0.031^{*}$ & 0.059 & & \\
\hline
\end{tabular}

U Mann-Whitney test, $p p$ value for comparing between the studied groups, $p_{1} p$ value for paired test or Wilcoxon signed-rank test for comparing between preand post-therapy, Group A cases, Group B control

*Statistically significant at $p \leq 0.05$ 
Table 5 Comparison between rater 1 and 2 in subjective assessment

\begin{tabular}{|c|c|c|c|c|c|c|c|c|}
\hline & \multicolumn{4}{|c|}{ Group A $(n=11)$} & \multicolumn{4}{|c|}{ Group B $(n=10)$} \\
\hline & ICC & $p$ & $(95 \% \mathrm{Cl})$ & LOA & ICC & $p$ & $(95 \% \mathrm{Cl})$ & LOA \\
\hline \multicolumn{9}{|l|}{ Anger pattern } \\
\hline Pre-therapy & $0.722^{*}$ & $0.002^{*}$ & $0.268-0.916$ & Moderate & $1.000^{*}$ & $<0.001^{*}$ & $1.000-1.000$ & Excellent \\
\hline Post-therapy & $0.962^{*}$ & $<0.001^{*}$ & $0.872-0.989$ & Excellent & $0.914^{*}$ & $<0.001^{*}$ & $0.694-0.978$ & Excellent \\
\hline \multicolumn{9}{|c|}{ Verbal disapproval } \\
\hline Pre-therapy & $0.974^{*}$ & $<0.001^{*}$ & $0.912-0.993$ & Excellent & $1.000^{*}$ & $<0.001^{*}$ & $1.000-1.000$ & Excellent \\
\hline Post-therapy & $0.845^{*}$ & $<0.001^{*}$ & $0.530-0.956$ & Good & $0.758^{*}$ & $0.003^{*}$ & $0.286-0.934$ & Good \\
\hline \multicolumn{9}{|c|}{ Verbal exclamation } \\
\hline Pre-therapy & $0.890^{*}$ & $<0.001^{*}$ & $0.660-0.969$ & Good & $0.862^{*}$ & $<0.001^{*}$ & $0.539-0.964$ & Good \\
\hline Post-therapy & $0.938^{*}$ & $<0.001^{*}$ & $0.798-0.983$ & Excellent & $0.882^{*}$ & $<0.001^{*}$ & $0.598-0.969$ & Good \\
\hline \multicolumn{9}{|c|}{ Wishful thinking } \\
\hline Pre-therapy & $0.917^{*}$ & $<0.001^{*}$ & $0.726-0.977$ & Excellent & $0.965^{*}$ & $<0.001^{*}$ & $0.866-0.991$ & Excellent \\
\hline Post-therapy & $0.771^{*}$ & $0.001^{*}$ & $0.377-0.932$ & Moderate & $0.949^{*}$ & $<0.001^{*}$ & $0.810-0.987$ & Excellent \\
\hline \multicolumn{9}{|l|}{ Warning speech } \\
\hline Pre-therapy & $0.693^{*}$ & $0.005^{*}$ & $0.231-0.905$ & Moderate & $1.000^{*}$ & $<0.001^{*}$ & $1.000-1.000$ & Excellent \\
\hline Post-therapy & 1.000 & $<0.001^{*}$ & $1.00-1.00$ & Excellent & $1.000^{*}$ & $<0.001^{*}$ & $1.000-1.000$ & Excellent \\
\hline
\end{tabular}

ICC intraclass correlation coefficient, LOA level of agreement, $p p$ value for comparing between rater 1 and 2

* Statistically significant at $p \leq 0.05$, Group A cases, Group $B$ control

Value of ICC below < 0.50: poor agreement, between 0.50 and < 0.75 : moderate agreement, between 0.75 and .90 : Good agreement, above 0.90 : excellent agreement

- Anger pattern, verbal exclamation, and warning speech showed a statistically significant increase in mean F0 between pre-therapy and post-therapy in the cases group.

- A statistically significant increase in the mean energy of anger pattern was found in the cases group between pre-therapy and post-therapy. While comparison of the scores of both studied groups post-therapy showed a statistically significant difference in mean energy of anger pattern, verbal disapproval, and warning speech.

\section{Discussion}

The results of the present study demonstrated a trend of improvement of the affective prosody of hearingimpaired children following intervention using the "prosody treatment program" [22] targeting receptive and expressive prosodic skills. A statistically significant improvement was found regarding the subjective scores of all the assessed emotions as shown in Table 4. The improvement of the subjective scores was accompanied by durational, f0, and intensity changes. The objective measures of the utterances post-intervention showed a pattern of changes in the cases group with similarities to those documented in literature that reported normal patterns of emotion production [26].

The findings of the current intervention regarding the expression of anger pattern showed that the improvement in the subjective scores was associated with a statistically significant increase in the mean duration and the mean energy of the utterance (Table 6). Expression of anger is reported in literature to be associated with an increase in mean energy and fundamental frequency [30, 31]. Bahgat et al. [26] found a positive correlation between anger pattern and frequency values but found no correlation with energy values in the production of normally developing Arabic-speaking children.

The statistically significant increase in the mean energy of verbal disapproval is in line with studies associating appropriate production of verbal disapproval with increase in the mean energy of the utterance [26]; therefore, justifying the subjective improvement of this pattern in the cases group.

Auditory perceptual improvement of the production of verbal exclamation (surprise) was accompanied objectively by a significant increase in mean duration and mean f0 among the cases group (Table 6). Studies demonstrated that expression of surprise is accompanied by an increase in mean frequency in normal production. No significant difference was found in the mean energy of the utterance. It is worth mentioning that Nakata et al. [9] mentioned the challenge facing children with $\mathrm{CI}$ in imitation of disappointment and surprise patterns.

Warning pattern demonstrated a statistically significant increase in mean energy and fo in preschool cases in comparison with the control group (Table 6). 
Table 6 Comparison between the two studied groups according to objective prosodic assessment

\begin{tabular}{|c|c|c|c|c|c|c|}
\hline \multicolumn{3}{|c|}{ Objective prosodic assessment } & \multirow[t]{2}{*}{ Group A $(n=11)$} & \multirow[t]{2}{*}{ Group B $(n=10)$} & \multirow[t]{2}{*}{ Test of sig. } & \multirow[t]{2}{*}{$p$} \\
\hline Anger pattern & Duration (s) & Pre-therapy & & & & \\
\hline & & Mean \pm SD & $0.69 \pm 0.28$ & $1.12 \pm 0.50$ & $\mathrm{t}=2.429^{*}$ & $0.029^{*}$ \\
\hline & & Post-therapy & & & & \\
\hline & & Mean \pm SD & $0.98 \pm 0.26$ & $1.09 \pm 0.61$ & $t=0.506$ & 0.622 \\
\hline & & $p_{1}$ & $0.025^{*}$ & 0.859 & & \\
\hline & Mean F0 (Hz) & Pre-therapy & & & & \\
\hline & & Mean \pm SD & $207.1 \pm 28.10$ & $211.2 \pm 27.64$ & $t=0.338$ & 0.739 \\
\hline & & Post-therapy & & & & \\
\hline & & Mean \pm SD & $223.5 \pm 25.81$ & $205.3 \pm 39.82$ & $t=1.253$ & 0.225 \\
\hline & & $p_{1}$ & $0.005^{*}$ & 0.667 & & \\
\hline & Mean energy (dB) & Pre-therapy & & & & \\
\hline & & Mean \pm SD & $52.92 \pm 6.36$ & $53.58 \pm 6.98$ & $t=0.227$ & 0.823 \\
\hline & & Post-therapy & & & & \\
\hline & & Mean \pm SD & $60.07 \pm 4.55$ & $52.64 \pm 5.27$ & $\mathrm{t}=3.464^{*}$ & $0.003^{*}$ \\
\hline & & $\mathbf{p}_{1}$ & $0.004^{*}$ & 0.657 & & \\
\hline \multirow[t]{15}{*}{ Verbal disapproval } & Duration (s) & Pre-therapy & & & & \\
\hline & & Mean \pm SD & $0.65 \pm 0.27$ & $0.70 \pm 0.38$ & $U=52.0$ & 0.833 \\
\hline & & Post-therapy & & & & \\
\hline & & Mean \pm SD & $1.02 \pm 0.53$ & $0.86 \pm 0.52$ & $U=43.0$ & 0.398 \\
\hline & & $p_{1}$ & $0.026^{*}$ & 0.799 & & \\
\hline & Mean F0 (Hz) & Pre-therapy & & & & \\
\hline & & Mean \pm SD & $215.5 \pm 32.0$ & $218.5 \pm 27.16$ & $t=0.233$ & 0.818 \\
\hline & & Post-therapy & & & & \\
\hline & & Mean \pm SD & $226.1 \pm 27.63$ & $212.8 \pm 27.68$ & $t=1.098$ & 0.286 \\
\hline & & $p_{1}$ & 0.343 & 0.451 & & \\
\hline & Mean energy (dB) & Pre-therapy & & & & \\
\hline & & Mean \pm SD & $55.40 \pm 6.62$ & $54.25 \pm 7.40$ & $t=0.378$ & 0.710 \\
\hline & & Post-therapy & & & & \\
\hline & & Mean \pm SD & $58.51 \pm 5.47$ & $51.21 \pm 8.32$ & $t=2.398^{*}$ & $0.027^{*}$ \\
\hline & & $p_{1}$ & 0.193 & 0.053 & & \\
\hline \multirow[t]{14}{*}{ Verbal exclamation } & Duration (s) & Pre-therapy & & & $t=0.659$ & 0.520 \\
\hline & & Mean \pm SD & $1.16 \pm 0.48$ & $1.35 \pm 0.79$ & & \\
\hline & & Post-therapy & & & & \\
\hline & & Mean \pm SD & $1.92 \pm 0.47$ & $1.30 \pm 0.45$ & $t=3.056^{*}$ & $0.007^{*}$ \\
\hline & & $p_{1}$ & $0.008^{*}$ & 0.798 & & \\
\hline & Mean F0 (Hz) & Pre-therapy & & & & \\
\hline & & Mean \pm SD & $188.1 \pm 15.77$ & $202.6 \pm 26.79$ & $t=1.531$ & 0.142 \\
\hline & & Post-therapy & & & & \\
\hline & & Mean \pm SD & $206.5 \pm 17.77$ & $205.3 \pm 25.68$ & $t=0.119$ & 0.906 \\
\hline & & $p_{1}$ & $0.002^{*}$ & 0.694 & & \\
\hline & Mean energy $(\mathrm{dB})$ & Pre-therapy & & & & \\
\hline & & Mean \pm SD & $53.85 \pm 8.81$ & $52.21 \pm 9.15$ & $t=0.418$ & 0.680 \\
\hline & & Post-therapy & & & & \\
\hline & & Mean \pm SD & $54.97 \pm 6.41$ & $50.23 \pm 7.92$ & $t=1.516$ & 0.146 \\
\hline
\end{tabular}


Table 6 Comparison between the two studied groups according to objective prosodic assessment (Continued)

\begin{tabular}{|c|c|c|c|c|c|c|}
\hline \multicolumn{3}{|c|}{ Objective prosodic assessment } & \multirow{2}{*}{$\begin{array}{l}\text { Group A }(n=11) \\
0.683\end{array}$} & \multirow{2}{*}{$\begin{array}{l}\text { Group B }(n=10) \\
0.557\end{array}$} & \multirow[t]{2}{*}{ Test of sig. } & \multirow[t]{2}{*}{$p$} \\
\hline & & $p_{1}$ & & & & \\
\hline \multirow[t]{16}{*}{ Wishful thinking } & Duration (s) & Pre-therapy & & & & \\
\hline & & Mean \pm SD & $1.11 \pm 0.52$ & $2.03 \pm 0.97$ & $U=20.0^{*}$ & $0.014^{*}$ \\
\hline & & Post-therapy & & & & \\
\hline & & Mean \pm SD & $1.97 \pm 0.77$ & $1.27 \pm 0.42$ & $U=14.0^{*}$ & $0.004^{*}$ \\
\hline & & Mean difference & $\uparrow 0.86 \pm 1.0$ & $\downarrow 0.77 \pm 1.03$ & $U=10.0^{*}$ & $0.002^{*}$ \\
\hline & & $p_{1}$ & $0.014^{*}$ & $0.047^{*}$ & & \\
\hline & Mean FO $(\mathrm{Hz})$ & Pre-therapy & & & & \\
\hline & & Mean \pm SD & $203.8 \pm 25.61$ & $216.7 \pm 25.57$ & $t=1.158$ & 0.261 \\
\hline & & Post-therapy & & & & \\
\hline & & Mean \pm SD & $224.6 \pm 28.60$ & $213.9 \pm 35.60$ & $t=0.767$ & 0.452 \\
\hline & & $p_{1}$ & 0.054 & 0.783 & & \\
\hline & Mean energy (dB) & Pre-therapy & & & & \\
\hline & & Mean \pm SD & $53.06 \pm 5.52$ & $50.58 \pm 8.67$ & $t=0.791$ & 0.439 \\
\hline & & Post-therapy & & & & \\
\hline & & Mean \pm SD & $53.93 \pm 5.19$ & $49.78 \pm 6.81$ & $t=1.581$ & 0.130 \\
\hline & & $\mathrm{p}_{1}$ & 0.691 & 0.677 & & \\
\hline \multirow[t]{15}{*}{ Warning speech } & Duration (s) & Pre-therapy & & & & \\
\hline & & Mean \pm SD & $1.40 \pm 0.67$ & $1.19 \pm 0.66$ & $t=0.722$ & 0.479 \\
\hline & & Post-therapy & & & & \\
\hline & & Mean \pm SD & $1.91 \pm 0.50$ & $1.08 \pm 0.71$ & $\mathrm{t}=3.132^{*}$ & $0.005^{*}$ \\
\hline & & $p_{1}$ & 0.064 & 0.534 & & \\
\hline & Mean F0 $(\mathrm{Hz})$ & Pre-therapy & & & & \\
\hline & & Mean \pm SD & $195.1 \pm 17.98$ & $215.2 \pm 26.02$ & $t=2.043$ & 0.058 \\
\hline & & Post-therapy & & & & \\
\hline & & Mean \pm SD & $208.8 \pm 21.88$ & $213.5 \pm 33.94$ & $t=0.373$ & 0.714 \\
\hline & & $p_{1}$ & $0.010^{*}$ & 0.875 & & \\
\hline & Mean energy (dB) & Pre-therapy & & & & \\
\hline & & Mean \pm SD & $52.79 \pm 6.77$ & $53.43 \pm 6.58$ & $t=0.221$ & 0.828 \\
\hline & & Post-therapy & & & & \\
\hline & & Mean \pm SD & $54.09 \pm 5.21$ & $48.74 \pm 3.52$ & $t=2.726^{*}$ & $0.013^{*}$ \\
\hline & & $\mathrm{p}_{1}$ & 0.549 & $0.028^{*}$ & & \\
\hline
\end{tabular}

$t$ Student's $\mathrm{t}$ test, $p p$ value for comparing between the studied groups, $p_{1} p$ value for paired $\mathrm{t}$ test or Wilcoxon signed-rank test for comparing between pre and post, Group A cases, Group B control

*Statistically significant at $p \leq 0.05$

Warning patterns are normally characterized by an increase in the mean energy of the utterance in Arabic-speaking children [26], indicating an improvement in the production of this pattern as perceived by subjective assessment.

No statistically significant difference was found in the frequency of verbal disapproval, the intensity of verbal exclamation, or the frequency and intensity of wishful thinking even though these patterns demonstrated a significant improvement of their subjective scores. The normal development of these patterns is achieved older than 6 years of age, mature control of fundamental frequency is not normally achieved before the age of 7 years, and changes in acoustic parameters signaling prosody might be minimal even though auditory perception is significantly changed $[26,32]$.

The results of the present study demonstrated that the significant improvement in the subjective scores is associated with increased duration of the utterance. This is contradictory to other studies reporting that appropriate 
prosodic development is associated with a decrease in the mean duration as a result of the increase in the speech rate of children as their maturation progresses [26]. The significant increase in the mean duration detected post intervention in the current study is most probably attributed to the increase in the sentence length of children as evidenced by the significant increase in their total language age and verbal $I Q$, not due to an inappropriate decrease in their speech rate.

The objective results of the current study indicate the effect of prosodic training on improving motor learning resulting in better control of pitch production. Additionally, the increase in the mean energy of the utterances indicates better phonatory abilities resulting in the ability to apply different loudness levels and expand the loudness range of the utterances of $\mathrm{HI}$ children to accommodate different contexts.

Improvement of prosodic production of emotions indicates the improvement in the ability of $\mathrm{HI}$ children to perceive intonational contours characteristic for each emotion, and spontaneously produce or imitate these utterances using prosodic patterns comparable to normal expressive patterns of affect. These findings were further confirmed by the acoustic objective analysis.

The preschool section of the intervention program included songs and experimenting with musical instruments to increase awareness of children of rhythmic and pitch variations. The results of the current study are in agreement with the findings of the study by Torppa [33] which demonstrated the role of intervention using music and rhythm in the improvement of prosody, in particular stress and emotion production, concluding that musical activities are associated with better stress perception in addition to improved expressive linguistic skills.

The pattern of prosodic improvement in our study is also in line with the results of Klieve and Jeanes [16] who detected considerable improvement of prosodic skills of children with hearing impairment after a 10-week intervention program targeting prosodic perception skills such as affect, loudness, question and statement, chunking and stress and reported their effect on the improvement of prosodic production abilities.

The program was used in training the children using activities targeting the main prosodic features: pitch, loudness, and duration. The learned prosodic skills were applied to produce adequate prosodic cues namely stress, rhythm, and intonation. Focusing on specific parameters and neglecting others was avoided, because targeting an individual prosodic parameter and disregarding others has been discouraged to avoid negatively altering a child's phonological system [34].

No statistically significant difference was detected in the control group receiving the conventional treatment suggesting that it is unlikely that auditory and language rehabilitation alone would achieve prosodic improvement in such a short period of time as that achieved by the group that received the prosody intervention program.

The results of the present study lead to the conclusion that rehabilitation of prosody has an additional benefit to $\mathrm{HI}$ children and the deficient prosodic abilities of $\mathrm{HI}$ children are responsive to motor planning and amenable to change by incorporating prosodic cues in a framework of meaningful language-based contexts, resulting in improvement of their expressive prosodic skills.

A statistically significant increase in the verbal IQ of the cases group was found when comparing pre-therapy and post-therapy scores (Table 2). This could be explained by the effect of prosodic skills on the development of language leading to an increase in verbal intelligence. These findings were further confirmed by the significant improvement detected in the language skills of the cases group (Table 3). These results confirm the role of prosody in language acquisition and word learning [35]. This is supported by the pragmatic and syntactic functions of prosody, as prosody is thought to have a prominent interaction with the pragmatic aspect of language particularly by its role in conveying phrasal stress. Moreover, prosody facilitates word acquisition by means of aiding in speech segmentation $[36,37]$. The contribution of prosody to language acquisition could be explained by the prosodic bootstrapping hypothesis, which proposes that children make use of the suprasegmental features during syntactic development. Thus, prosody bootstraps the lexical and syntactic acquisition [38].

\section{Conclusion}

The results presented indicate that prosodic intervention improves the production of emotions in children with SNHL. Moreover, prosody training may have a role in accelerating language acquisition in $\mathrm{HI}$ children. Therefore, the assessment of prosodic skills in HI children to detect their prosodic deficits is warranted in order to incorporate prosodic training in their speech and language rehabilitation plans. Conducting longitudinal studies with larger samples is suggested to detect if the acquired skills were maintained or further developed.

\section{Limitations}

The results of the current study should be interpreted with caution as the study is subject to some limitations that could be addressed in future research. The study sample size was small, replication of the study on a larger sample is recommended. The studied groups did not include normal hearing children which might have been beneficial. 


\section{Abbreviations}

Cl: Cochlear implant; F0: Fundamental frequency; HA: Hearing aids; $\mathrm{HI}$ : Hearing impairment; IQ: Intelligence quotient; SNHL: Sensorineural hearing loss

\section{Acknowledgements}

Not applicable.

\section{Authors' contributions}

OS contributed by designing the analysis and interpreting the data. RA contributed to writing the manuscript. SI contributed by data collection, providing the intervention, writing the paper, and interpreting the results. $\mathrm{NH}$ contributed to writing the manuscript and interpreting the data. All authors read and approved the final manuscript.

\section{Funding}

None.

\section{Availability of data and materials}

The datasets used and/or analyzed during the current study are available from the corresponding author on reasonable request.

\section{Declarations}

\section{Ethics approval and consent to participate}

The research was conducted after approval of the ethics committee of the Faculty of Medicine, Alexandria University (IRB No: 00012098). Informed verbal consent was taken from parents and/or legally caring surrogate of al children participating in the study and this was approved by the ethics committee.

\section{Consent for publication}

Not applicable

\section{Competing interests}

Dr Rania M. Abdou is a co-author of this study and editorial board member of the journal. She declares a competing interest for this submission. She has not handled this manuscript. The rest of the authors have no conflict of interest to declare.

\section{Author details}

${ }^{1}$ Audiovestibular Unit, Department of Otorhinolaryngology, Faculty of Medicine, Alexandria University, Champollion Street, 21131 Alexandria, Egypt. ${ }^{2}$ Unit of Phoniatrics, Department of Otorhinolaryngology, Faculty of Medicine, Alexandria University, Champollion Street, Alexandria 21131, Egypt.

\section{Received: 5 February 2021 Accepted: 19 May 2021}

\section{Published online: 14 June 2021}

\section{References}

1. Hargrove PM, McGarr NS (1993) A transdisciplinary model for prosody applied to the teaching of clinical populations. ESCA Workshop on Prosody, Lund

2. Peppé SJE (2009) Why is prosody in speech-language pathology so difficult? Int J Speech-Lang Pathol 11(4):258-271. https://doi.org/10.1080/1754 9500902906339

3. Couper-Kuhlen E (1986) Functions of intonation. An introduction to english prosody. Edward Arnold, London, pp 110-120

4. Paul R, Augustyn A, Klin A, Volkmar FR (2005) Perception and production of prosody by speakers with autism spectrum disorders. J Autism Dev Disord 35(2):205-220. https://doi.org/10.1007/s10803-004-1999-1

5. Bryant G, Barrett HC (2008) Vocal emotion recognition across disparate cultures. J Cogn Cult 8(1-2):135-148. https://doi.org/10.1163/156770908X2 89242

6. Wright A, Saxena S, Sheppard SM, Hillis AE (2018) Selective impairments in components of affective prosody in neurologically impaired individuals. Brain Cogn 124:29-36. https://doi.org/10.1016/j.bandc.2018.04.001

7. Dammeyer J (2010) Psychosocial development in a Danish population of children with cochlear implants and deaf and hard-of-hearing children. J Deaf Stud Deaf Educ 15(1):50-58. https://doi.org/10.1093/deafed/enp024
8. Percy-Smith L, Jensen JH, Cayé-Thomasen P, Thomsen J, Gudman M, Lopez AG (2008) Factors that affect the social well-being of children with cochlear implants. Cochlear Implants Int 9(4):199-214. https://doi.org/10.1179/cim.2 008.9.4.199

9. Nakata T, Trehub SE, Kanda Y (2012) Effect of cochlear implants on children's perception and production of speech prosody. J Acoustical Soc Am 131(2):1307-1314. https://doi.org/10.1121/1.3672697

10. Paquette S, Ahmed GD, Goffi-Gomez MV, Hoshino ACH, Peretz I, Lehmann A (2018) Musical and vocal emotion perception for cochlear implants users. Hear Res 370:272-282. https://doi.org/10.1016/j.heares.2018.08.009

11. Most T, Michaelis H (2012) Auditory, visual, and auditory-visual perceptions of emotions by young children with hearing loss versus children with normal hearing. J Speech, Language, Hearing Res 55(4):1148-1162. https:// doi.org/10.1044/1092-4388(2011/11-0060)

12. Most T, Aviner C (2009) Auditory, visual, and auditory-visual perception of emotions by individuals with cochlear implants, hearing aids, and normal hearing. J Deaf Stud Deaf Educ 14(4):449-464. https://doi.org/10.1093/dea fed/enp007

13. Chin SB, Bergeson TR, Phan J (2012) Speech intelligibility and prosody production in children with cochlear implants. J Commun Disord 45(5):355366. https://doi.org/10.1016/j.jcomdis.2012.05.003

14. Wang D, Trehub S, Volkova A, van Lieshout P (2013) Child implant users' imitation of happy- and sad-sounding speech. Front Psychol 4:351

15. Chatterjee M, Kulkarni AM, Siddiqui RM, Christensen JA, Hozan M, Sis JL, Damm SA (2019) Acoustics of emotional prosody produced by prelingually deaf children with cochlear implants. Front Psychol 10:2190. https://doi. org/10.3389/fpsyg.2019.02190

16. Klieve S, Jeanes R (2001) Perception of prosodic features by children with cochlear implants: is it sufficient for understanding meaning differences in language? Deaf Edu Inter 3(1):15-37. https://doi.org/10.1179/146431501 790561061

17. Friedman M (1985) Remediation of intonation contours of hearing-impaired students. J Commun Disord 18(4):259-272. https://doi.org/10.1016/00219924(85)90003-6

18. Sztahó D, Kiss G, Vicsi K (2018) Computer based speech prosody teaching system. Comput Speech Lang 50:126-140. https://doi.org/10.1016/j.csl.201 7.12 .010

19. Chen JKC, Chuang AYC, McMahon C, Hsieh JC, Tung TH, Li LPH (2010) Music training improves pitch perception in prelingually deafened children with cochlear implants. Pediatrics. 125(4):e793-e800. https://doi.org/10.1542/ peds.2008-3620

20. Bassiouny SE, Saleh MM, Elrefaie DA, Girgis MS (2017) Using music therapy in (re) habilitation of prelingual deaf cochlear implant children. Biom J Sci Tech Res 1(1):105-110

21. Good A, Gordon KA, Papsin BC, Nespoli G, Hopyan T, Peretz I, Russo FA (2017) Benefits of music training for perception of emotional speech prosody in deaf children with cochlear implants. Ear Hear 38(4):455-464. https://doi.org/10.1097/AUD.0000000000000402

22. Rothstein JA (2013) Prosody treatment program. Linguisystems, Austin

23. Albert ML, Sparks RW, Helm NA (1973) Melodic intonation therapy for aphasia. Arch Neurol 29(2):130-131. https://doi.org/10.1001/archneur.1973. 00490260074018

24. Ramig LO, Pawlas AA, Countryman S (1995) The lee Silverman voice treatment: a practical guide for treating the voice and speech disorders in Parkinson disease. National Center for Voice and Speech, lowa city

25. Strand EA, Stoeckel R, Baas B (2006) Treatment of severe childhood apraxia of speech: a treatment efficacy study. J Med Speech Lang Pathol 14(4):297307

26. Bahgat M, Khaled A, Aziz A, Osman D, Azab S (2012) Studying some elicited verbal prosodic patterns in Egyptian children. Egyptian J Otolaryngol 28(3): 242-250

27. Kotby MNKA, Baraka M, Rifaie N, El Shobary A (1995) Language testing of Arabic speaking children. In: Proceeding of the XXIII world congress of the International Association of Logopedics and Phoniatrics

28. Melika L (1998) The Stanford Binet intelligence scale. In: Arabic Examiner's handbook, 4th edn. Dar El-Maref Publishing, Cairo

29. Johnstone T, Scherer KR (2000) Vocal communication of emotion. In: Handbook of emotions, vol 2, pp 220-235

30. Sobin C, Alpert M (1999) Emotion in speech: the acoustic attributes of fear, anger, sadness, and joy. J Psycholinguist Res 28(4):347-365. https://doi.org/1 0.1023/A:1023237014909 
31. Gangamohan P, Kadiri SR, Yegnanarayana B (2016) Analysis of emotional speech-a review. In: Esposito A, Jain LC (eds) Toward robotic socially believable behaving systems-volume I. Springer, pp 205-238. Springer international publishing, Switzerland

32. Russell S, Laures-Gore J, Patel R (2010) Treating expressive aprosodia: a case study. J Med Speech-Language Pathol 18(4):115-120

33. Torppa R, Faulkner A, Laasonen M, Lipsanen J, Sammler D (2020) Links of prosodic stress perception and musical activities to language skills of children with cochlear implants and normal hearing. Ear Hear 41(2):395-410. https://doi.org/10.1097/AUD.0000000000000763

34. O'Halpin R (2001) Intonation issues in the speech of hearing impaired children: analysis, transcription and remediation. Clin Linguistics Phonetics 15(7):529-550. https://doi.org/10.1080/02699200110078131

35. Prieto P, Esteve-Gibert N (2018) The development of prosody in first language acquisition, pp 18-100. John Benjamins, Netherlands

36. Gerken L, McGregor K (1998) An overview of prosody and its role in normal and disordered child language. Am J Speech-Language Pathol 7(2):38-48. https://doi.org/10.1044/1058-0360.0702.38

37. Johnson EK, Jusczyk PW (2001) Word segmentation by 8-month-olds: when speech cues count more than statistics. J Mem Lang 44(4):548-567

38. Landau B, Gleitman LR, Landau B (2009) Language and experience: evidence from the blind child. Harvard University Press, Cambridge

\section{Publisher's Note}

Springer Nature remains neutral with regard to jurisdictional claims in published maps and institutional affiliations.

\section{Submit your manuscript to a SpringerOpen ${ }^{\circ}$ journal and benefit from:}

- Convenient online submission

- Rigorous peer review

- Open access: articles freely available online

- High visibility within the field

- Retaining the copyright to your article 